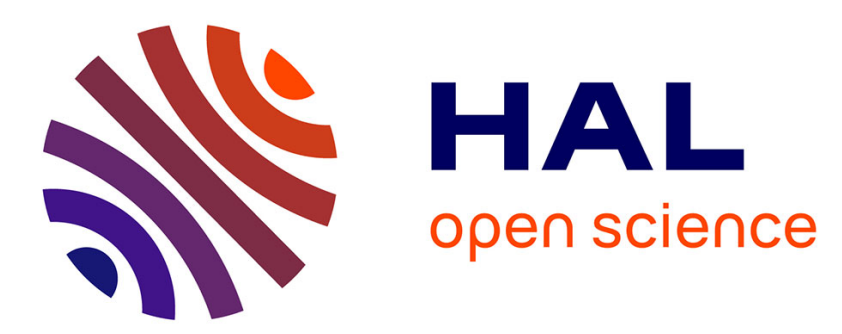

\title{
Influence of monomer-micelle exchange on micelle diffusion
}

\author{
P. Turq, M. Drifford, M. Hayoun, A. Perera, J. Tabony
}

\section{To cite this version:}

P. Turq, M. Drifford, M. Hayoun, A. Perera, J. Tabony. Influence of monomer-micelle exchange on micelle diffusion. Journal de Physique Lettres, 1983, 44 (12), pp.471-479. 10.1051/jphyslet:019830044012047100 . jpa-00232219

\section{HAL Id: jpa-00232219 https://hal.science/jpa-00232219}

Submitted on 1 Jan 1983

HAL is a multi-disciplinary open access archive for the deposit and dissemination of scientific research documents, whether they are published or not. The documents may come from teaching and research institutions in France or abroad, or from public or private research centers.
L'archive ouverte pluridisciplinaire HAL, est destinée au dépôt et à la diffusion de documents scientifiques de niveau recherche, publiés ou non, émanant des établissements d'enseignement et de recherche français ou étrangers, des laboratoires publics ou privés. 
Classification

Physics Abstracts

$64.70 \mathrm{~J}-78.35-82.40-82.70$

\title{
Influence of monomer-micelle exchange on micelle diffusion
}

\author{
P. Turq $\left({ }^{*}\right)$, M. Drifford $\left({ }^{* *}\right)\left({ }^{+}\right)$, M. Hayoun $(*)$, A. Perera $\left({ }^{* *}\right)$ and J. Tabony $\left({ }^{* *}\right)$ \\ (*) Laboratoire d'Electrochimie, Université Pierre et Marie Curie, \\ ERA 310, 4, Place Jussieu, 75005 Paris, France \\ (**) Département de Physico-Chimie, CEN-Saclay, 91191 Gif sur Yvette Cedex, France
}

(Reçu le 19 janvier 1983, révisé le 22 avril, accepté le 23 avril 1983)

\begin{abstract}
Résumé. - L'influence de l'échange monomères-micelles sur les propriétés de transport des systèmes micellaires à forte concentration micellaire critique est mise en évidence. La diffusion de la lumière de l'octanoate de sodium et de l'hexylsulfate de sodium est analysée à partir d'un nouveau modèle cinétique en tenant compte des interactions micelles-micelles.
\end{abstract}

\begin{abstract}
For micellar systems having a high critical micellar concentration, it is shown that the monomer-micelle exchange makes a significant contribution to the diffusion processes. Practical examples are micelles of sodium octanoate and sodium hexyl sulphate. Their diffusion coefficients are analysed in the framework of a kinetic model which includes the effect of intermicellar interactions.
\end{abstract}

\section{Introduction.}

Of the different methods for measuring diffusion coefficients in micellar systems, dynamic light scattering differs from the others [1-3] in observing the micelle directly. The observed diffusion coefficient hence depends only on the micelles and their mutual interactions and should not contain a contribution from the diffusion of the monomers. This is the case in micellar systems with a low critical micellar concentration (below $0.1 \mathrm{M}$ ), such as S.D.S. [4] and C.T.A.B. [5]. However by assuming a dynamical effect during the expulsion of a monomer by the micelle, Phillies [6] has recently introduced the idea of a coupling of the micelle diffusion to that of monomer-micelle exchange and has shown that for micelles having a high c.m.c. the diffusion coefficient measured by light scattering may be perturbed by that of the monomer.

In this paper, the effect of monomer-micelle exchange is treated in terms of reaction rates rather than dynamics; it takes into account intermicellar interactions. A similar treatment for the kinetics of aggregation processes has been proposed by Evans et al. [7]. They developed two limiting cases depending upon whether the micellar aggregation has fast or slow reaction rates. Because intermicellar interactions, are not considered, the fast reaction rate theory only applies at low micellar concentrations when these interactions are weak.

The analysis proposed in this paper is applied to the concentration variation of the diffusion coefficient of micellar solutions of sodium hexyl sulfate (S.H.S.) and sodium octanoate (S.Oct.).

$\left({ }^{+}\right)$To whom correspondence should be addressed. 


\section{Monomer-micelle equilibrium.}

Aniansson et al. [8] have treated the formation of micelles in terms of two relaxation processes having different time constants.

The first relaxation process is fast and changes the micellar size by gain or loss of a monomer. A dynamic equilibrium is obtained yielding a distribution of micellar sizes around $\bar{N}$ given by :

$$
\mathrm{m}+\mathrm{m}_{N-1} \underset{k_{2}}{\stackrel{k_{1}}{\rightleftarrows}} \mathrm{M}
$$

where $\mathrm{M}$ represents the micelle (species 1), $\mathrm{m}$ the monomer (species 2 ) and $N$ the aggregation number.

$$
\begin{array}{ll}
k_{1} \sim 10^{+7} \mathrm{~s}^{-1} & \text { for S.D.S. } \\
k_{1} \sim 10^{+9} \mathrm{~s}^{-1} & \text { for S.H.S. and S.Oct. }
\end{array}
$$

The second relaxation process ensures equilibrium between the monomer concentration and the total number density of micelles. This formation-dissolution process can be written as

$$
N \mathrm{~m} \underset{k_{\mathrm{m}}}{\stackrel{k_{\mathrm{M}}}{\rightleftarrows}} \mathbf{M} \text {. }
$$

For micelles with a long aliphatic chain (S.D.S.-C.T.A.B.), this process is fast $\left(10^{-3} \mathrm{~s}\right.$ to $\left.1 \mathrm{~s}\right)$. But for small micelles, with a large c.m.c. like S.H.S. and S.Oct., the relaxation time, $k_{\mathrm{M}}^{-1}$ is very short $\left(10^{-8}\right.$ to $\left.10^{-9} \mathrm{~s}\right)$. Thus the characteristic times of the two processes (monomer exchange and formation dissolution) are comparable [9].

In dynamic light scattering measurements, one observes diffusion over distances comparable to the wavelength of light, during a time $\tau_{D}=\left(2 D q^{2}\right)^{-1}$ where $D$ is the diffusion coefficient of the micelle and $q$ the scattering vector. For S.D.S. with $\lambda=5,145 \AA$ at a scattering angle of $90^{\circ}, \tau_{\mathrm{D}}$ is approximately $10 \mu \mathrm{s}$. For S.H.S. and sodium octanoate, under the same conditions, $\tau_{D} \sim 5 \mu$ s. The residence time of a monomer in the micelle $\left(\tau_{R}\right)$ is $N / K_{m}$. For S.D.S. $\tau_{R} \sim 6.4 \mu \mathrm{s}$ whilst for S.H.S. and sodium octanoate it is $20 \mathrm{~ns}$. Hence during the diffusion time, $\tau_{D}$, each monomer is replaced many times, and for S.H.S. and octanoate each micelle breaks up approximately 250 times. Also for the latter, the c.m.c. is high $\sim 0.5 \mathrm{M}$, and for concentrations just above it $(C$-c.m.c. $\approx 0.05 \mathrm{M})$ there are 200 times more monomers than micelles. Here the Brownian motion as measured by light scattering may be modified by monomer-micelle exchange reactions.

\section{Theory.}

In dynamic light scattering, the micellar diffusion coefficient is obtained from the analysis of the relaxation processes for the concentration fluctuation. This is essentially a diffusional process. For a micelle undergoing chemical exchange with monomers, the latter will affect the diffusion coefficient in the following way : the micelle can be considered as diffusing on its own or can be decomposed into fast diffusing monomers which reform into another observable micelle. The net effect of the two-state diffusion will be an increase in the effective micellar diffusion coefficient. A quantitative treatment of this effect can be obtained using a normal-mode analysis [10] we have initially treated an uncharged micelle undergoing monomer-micelle exchange and having short range intermicellar interactions (hard sphere collisions). We shall subsequently discuss the effect of charge on micellar diffusion in a future publication.

3.1 UNCHARGED OR HIGHLY SCREENED MICELLES. - The kinetics of micellization may be characterized by the reaction of equation 2 . The reaction order with respect to the monomer is 
$v(1 \leqslant v \leqslant N)$ but ịs one with respect to the micellar decomposition. A value of $v>1$ can be interpreted as a cooperative phenomenon involving some premicellar clusters.

The continuity equations for the diffusion of species 1 and 2 are :

$$
\begin{aligned}
& \frac{\partial C_{1}}{\partial t}+\nabla \mathbf{J}_{1}=k_{\mathrm{M}} C_{2}^{v}-k_{\mathrm{m}} C_{1}, \\
& \frac{\partial C_{2}}{\partial t}+\nabla \mathbf{J}_{2}=N\left(-k_{\mathrm{M}} C_{2}^{v}+k_{\mathrm{m}} C_{1}\right),
\end{aligned}
$$

where $C_{i}$ is the number density and $J_{i}$ is the local flux.

The quantity $k_{\mathrm{M}} C_{2}^{v}$ represents the formation of micelles and $k_{\mathrm{m}} C_{1}$ is the rate of break up of aggregates.

The equilibrium condition for the average values of concentration is $k_{\mathrm{M}}\left(C_{2}^{0}\right)^{v}-k_{\mathrm{m}} C_{1}^{0}=0$.

The local instantaneous concentration $C_{i}$ is the sum of an average and a fluctuating term

$$
C_{i}=C_{i}^{0}+\delta C_{i} \text {. }
$$

Assuming that the two species 1 and 2 satisfy Fick's law

$$
\mathbf{J}_{i}=-D_{i} \nabla C_{i} .
$$

$D_{i}$ is the diffusion constant of the $i$-th species.

Substitution of (4) and (5) into (3) leads to the linearized equation :

$$
\begin{aligned}
\frac{\partial}{\partial t} \delta C_{1}-D_{1} \nabla^{2} \delta C_{1} & =-k_{\mathrm{m}}\left[\delta C_{1}-N \frac{C_{1}^{0}}{C_{2}^{0}} \delta C_{2}\right] \\
\frac{\partial}{\partial t} \delta C_{2}-D_{2} \nabla^{2} \delta C_{2} & =N k_{\mathrm{m}}\left[\delta C_{1}-N \frac{C_{1}^{0}}{C_{2}^{0}} \delta C_{2}\right] .
\end{aligned}
$$

The standard methods involving Fourier transformation on the space variable and Laplace transformation in time yield the general equations :

$$
\begin{gathered}
\delta C_{1}(q, s)\left[s+q^{2} D_{1}+k_{\mathrm{m}}\right]-N k_{\mathrm{m}} \frac{C_{1}^{0}}{C_{2}^{0}} \delta C_{2}(q, s)=\delta C_{1}(q, 0), \\
\delta C_{2}(q, s)\left[s+q^{2} D_{2}+N^{2} k_{\mathrm{m}} \frac{C_{1}^{0}}{C_{2}^{0}}\right]-N k_{\mathrm{m}} \delta C_{1}(q, s)=\delta C_{2}(q, 0) .
\end{gathered}
$$

The normal modes are given by the roots of the determinant of equation 7 .

When $q^{2} D_{i} \gg k_{\mathrm{m}}$, each species diffuses with its proper diffusion constant and, in this case, the light scattering sees only the Brownian motion of the micelles. When $q^{2} D_{i} \ll k_{\mathrm{m}}$ with fast chemical exchange, one root tends to the effective diffusion coefficient, which is given by the expression :

$$
D_{\mathrm{eff}}=\frac{D_{1} N v \frac{C_{1}^{0}}{C_{2}^{0}}+D_{2}}{1+N v \frac{C_{1}^{0}}{C_{2}^{0}}} .
$$

The micelle-monomers equilibrium is instantaneously established at $t \rightarrow 0$ and remains constant as both micelles and monomers diffuse. 
If the monomers are assumed to be ideal and the micelles to undergo short-range interactions $[11,12]$.

$$
D_{1}=D_{1}^{0}\left(1+\delta_{1}\right)
$$

where $\delta_{1}$ is the hydrodynamic correction which can be evaluated from micelle-micelle distribution functions by means of Oseen's tensor [13]. $D_{1}^{0}$ is the hydrodynamic diffusion coefficient of the micelles without interactions.

$$
\delta_{1}=1-\frac{C_{1} G_{11}}{1+C_{1} G_{11}}
$$

at $q \rightarrow 0$

$$
G_{11}=\int_{0}^{\infty} 4 \pi r^{2}\left[g_{11}(r)-1\right] \mathrm{d} r
$$

where $g_{11}(r)$ is the micelle-micelle pair distribution function [14-16]. In the first order for hard sphere micelles

$$
D_{1}=D_{1}^{0}(1+k \phi),
$$

where $\phi$ is the volume fraction of the micelle and $k$ varies from 1.5 to $2.0[10,13]$. The most noticeable result is that equation 8 predicts the existence of a minimum for the variation of $D_{\text {eff }}$ with the total surfactant concentration (or with the volume fraction of the micelle). The physical significance of this minimum is simple : at low concentration, in the vicinity of the c.m.c., the diffusion coefficient is increased by the monomer-micelle exchange reaction, and in the high concentration region by direct micelle-micelle interactions.

The experimental study of S.H.S. and S.Oct. where : $k_{\mathrm{m}}\left(10^{+9} \mathrm{~s}^{-1}\right) \gg D q_{2}\left(0.5 \times 10^{+6} \mathrm{~s}^{-1}\right)$ is a good test to illustrate this large effect.

\section{Experimental.}

Sodium octanoate, (S.Oct.) $\left(\mathrm{CH}_{3}-\left(\mathrm{CH}_{2}\right)_{7} \mathrm{COONa}\right)$ was purified and recrystallized as described in [17]. Sodium hexylsulfate, (S.H.S.) $\left(\mathrm{CH}_{3}-\left(\mathrm{CH}_{2}\right)_{5}-\mathrm{OSO}_{3} \mathrm{Na}\right)$ was purchased from Eastman Kodak and was purified by recrystallizing three times from ethyl alcohol and drying in vacuum at $30^{\circ} \mathrm{C}$.

The c.m.c. were determined by conductivity measurements at $25^{\circ} \mathrm{C}$ and the values obtained are :

$$
\begin{aligned}
& \text { c.m.c.(s.Oct.) }_{\text {. }} \simeq 0.40 \mathrm{M} \pm 0.02 \mathrm{M} \\
& \text { c.m.c. } \\
& \text { (S.H.S.) }
\end{aligned}
$$

The apparatus, cell filling method, homodyne detection and cumulant data analysis of the autocorrelation functions were identical with those previously used for the study of polyelectrolytes [18] and micelles [19].

The low scattering intensity of these " small micelles " requires both particular experimental care and an accurate cumulant data analysis. Values of both the average decay rate $\bar{\Gamma}$ and the polydispersity factor $\frac{\mu^{2}}{\bar{\Gamma}^{2}}$ were calculated from two fittings (linear and quadratic) of the data versus $\bar{\Gamma}_{i} \tau_{\max }$ 


\section{Results and discussion.}

A typical plot of the effective diffusion coefficient of S.H.S. against the micellar concentration, $C$-c.m.c., is shown in figure 1 . The same behaviour is observed for S.Oct. [19]. The effective diffusion coefficient decreases slowly in an approximately linear way with decreasing micelle concentration until a value of $C$ just above the c.m.c. below which it increases dramatically. This increase is rather less pronounced for S.Oct.

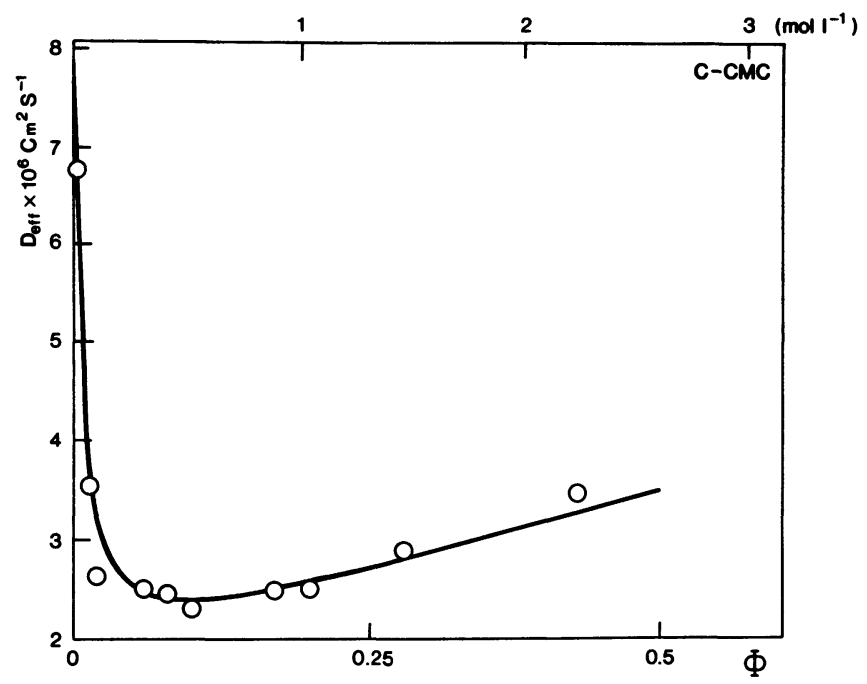

Fig. 1. - Effective diffusion coefficient $D_{\text {eff }}$ versus the volume fraction $\phi$ of S.H.S. $O$ : experimental data. Full line : theoretical curve with $D_{1}^{0}=1.74 \times 10^{-6} \mathrm{~cm}^{2} / \mathrm{s}, D_{2}^{0}=8 \times 10^{-6} \mathrm{~cm}^{2} / \mathrm{s}, \bar{N}=20, R_{\mathrm{H}}=11.5 \AA$, $k=2$.

$D_{\text {eff }}$ shows a minimum and this is qualitatively in agreement with the theory (Eq. 8). Near the c.m.c., the effective diffusion coefficient measured by light scattering is very high and close to the monomer diffusion coefficient. At high concentration the variation of the micellar diffusion coefficient is influenced by the micelle-micelle interaction. The slope is positive indicating a predominantly hard-sphere potential with eventually a small repulsive contribution due to the degree of ionization of the micelles.

To discriminate between conventional chemical reaction and diffusional micelle-monomer exchange, we have shown that the linewidth $\bar{\Gamma}$ is linear in $q^{2}$ with $\bar{\Gamma} \rightarrow 0$ as $q^{2} \rightarrow 0$ near the c.m.c. for S.Oct. [19]. This effect is diffusive and can be resolved by normal-mode analysis.

The micellar solutions of SHS and S.Oct. have a very large c.m.c. and we can consider that electrostatic interactions are screened by the counter-ions and free monomers. We then applied the theory for non charged micelles (Eq. 8) to experimental data.

The evaluation of the volume fraction of micelle, $\phi$, is difficult because it is necessary to know the micellar radius and the aggregation number :

$$
\begin{aligned}
& \phi=\frac{4 \pi R^{3}}{3} N_{\mathrm{A}} C_{1}^{0} \\
& \phi=v_{1} C_{1}^{0},
\end{aligned}
$$

where $v_{1}$ is the partial molar volume of micelle.

With a constant free monomer concentration $C_{2}^{0}=$ c.m.c. and taking $v \simeq N$, which means 
that the micelle aggregation is spontaneous with a mean aggregation number of $N$ monomers, the equation 8 can be written :

$$
D_{\text {eff }}=\frac{\frac{D_{1}^{0} N^{2} \phi(1+k \phi)}{v_{1} \times \text { c.m.c. }}+D_{2}^{0}}{1+\frac{N^{2} \phi}{v_{1} \times \text { c.m.c. }}} .
$$

The minimum in $D_{\text {eff }}$ is directly proportional to $\sqrt{\text { c.m.c. }}$ and inversely proportional to the square of the aggregation number. Micelles of S.H.S. and S.Oct. which have low aggregation numbers $(N \simeq 20)$ and large c.m.c. $(\sim 0.5 \mathrm{M})$ are good experimental subjects for the influence monomer-micelle chemical exchange since they should show a clear minimum in $D_{\text {eff }}$ with increasing concentration.

5.1 STUDY OF S.H.S. $-D_{1}^{0}$ was determined by the extrapolation of the linear portion of the experimental values of $D$ obtained at high concentration. We find $D_{1}^{0} \simeq 1.75 \times 10^{-6} \mathrm{~cm}^{2} / \mathrm{s}$. $D_{2}^{0}$ was obtained from conductivity measurements at low concentration :

$$
D_{2}^{0} \simeq 8 \times 10^{-6} \mathrm{~cm}^{2} / \mathrm{s} \text {. }
$$

$N$ is more difficult to obtain experimentally from light scattering. Because the dependence of the aggregation number on concentration could be important for these small micelles [19], the extrapolation of classical parameters such as the Rayleigh ratio $K c / R \theta$ to the c.m.c. is not valid. We took $\bar{N}=20+2[6,20]$. $\phi$ and $v_{1}$ were obtained from equation 13 and from the hydrodynamic radius $R_{\mathrm{H}}^{0}(11.5 \AA)$ derived from the Einstein-Stokes relation, $R_{\mathrm{H}}^{0}=k T / 6 \pi \eta D_{1}^{0}$ where $\eta$ is the viscosity of the solution at the c.m.c. $(\eta=1.138 \mathrm{cP})$.

The values of $D_{1}^{0}, N$, and $R_{\mathrm{H}}^{0}$ are in agreement with Phillies' data (6) which were deduced from geometrical considerations correlated to S.D.S.

5.2 STUDY OF S.OCT. - All parameters used in equation 14 have been obtained by both neutron scattering [21] and light scattering [19] :

$$
D_{1}^{0}=1.95 \times 10^{-6} \mathrm{~cm}^{2} / \mathrm{s}, \quad D_{2}^{0}=6 \times 10^{-6} \mathrm{~cm}^{2} / \mathrm{s} .
$$

$N$ varies between 12 and at the c.m.c. to 26 at $1.8 \mathrm{M}$.

We used the same values of $\phi_{\mathrm{HS}}$ as in neutron and light scattering studies. This is the sum of the partial volume of the S.Oct. molecule [22] and a water hydration shell of about 8-9 water molecules per monomer unit. In this case, the volume fraction of the micelle in solution is considered to be independent of the aggregation number of the micelle.

Table I shows the values of $C$-c.m.c., $\phi_{\mathrm{HS}}$ and $D_{\mathrm{eff}}$ for S.D.S. and S.Oct.

Figure 1 shows good agreement between experimental values $(\oplus)$ and the theoretical behaviour (full line) for the effective diffusion coefficient of S.H.S. as a function of $\phi_{\text {HS. The best fit is }}$ obtained with $k=2$. When the dependence of the aggregation number on concentration is low ( $D_{0}^{1}$ is constant), $k$ corresponds to the hard-sphere potential. For a variation of $N$ with $\phi, D_{0}^{1}$ varies approximately as $N^{-1 / 3}$. The experimental value of $k$ results from the combination of two effects interactions (hard-sphere plus electrostatic) and $\phi$-dependence of $N$, and it is not possible to draw any conclusions from the value for $k$.

Similarly, figure 2 shows the theoretical curve and experimental data for S.Oct. We have used the concentration dependence of the aggregation number and all the parameters are known. The best fit was obtained for $k \simeq 1.7$. In this case, if we use a constant aggregation number of 25 , we do not obtain a good agreement with experiment. However, the concentration dependence of $D_{\text {eff }}$ cannot be used to determine the aggregation number. 
Table I. - Experimental values of effective diffusion coefficient $D_{\text {eff }}$ for different volume fraction $\phi$ of S.H.S. and S.Oct.

S.H.S.

S.Oct.

\begin{tabular}{|c|c|c|c|c|c|}
\hline $\begin{array}{c}C \text {-c.m.c. : }(\mathrm{M} / \mathrm{l}) \\
(\overline{c . m . c .}=0.57 \mathrm{M})\end{array}$ & $\begin{array}{c}\phi=\phi_{\mathrm{HS}} \\
\bar{N}=20 R_{\mathrm{H}}=11.5 \AA\end{array}$ & $\begin{array}{c}D_{\text {eff }} \\
\times 10^{6}\left(\mathrm{~cm}^{2} / \mathrm{s}\right)\end{array}$ & $\begin{array}{c}C \text {-c.m.c. } \\
\mathrm{M} / 1\end{array}$ & $\phi_{\mathrm{HS}}[17]$ & $\begin{array}{c}D_{\text {eff }} \\
\times 10^{6}\left(\mathrm{~cm}^{2} / \mathrm{s}\right)\end{array}$ \\
\hline 0.01 & 0.002 & 6.8 & 0.2 & 0.06 & 2.7 \\
0.10 & 0.017 & 3.54 & 0.4 & 0.12 & 2.6 \\
0.13 & 0.02 & 2.63 & 0.6 & 0.18 & 2.5 \\
0.30 & 0.06 & 2.51 & 0.8 & 0.24 & 2.7 \\
0.40 & 0.08 & 2.47 & 1.0 & 0.30 & 3.0 \\
0.56 & 0.10 & 2.32 & 1.2 & 0.35 & 3.1 \\
0.88 & 0.17 & 2.50 & 1.4 & 0.41 & 3.3 \\
1.10 & 0.20 & 2.52 & & & \\
1.52 & 0.28 & 3.50 & & & \\
2.27 & 0.43 & & & \\
\hline
\end{tabular}

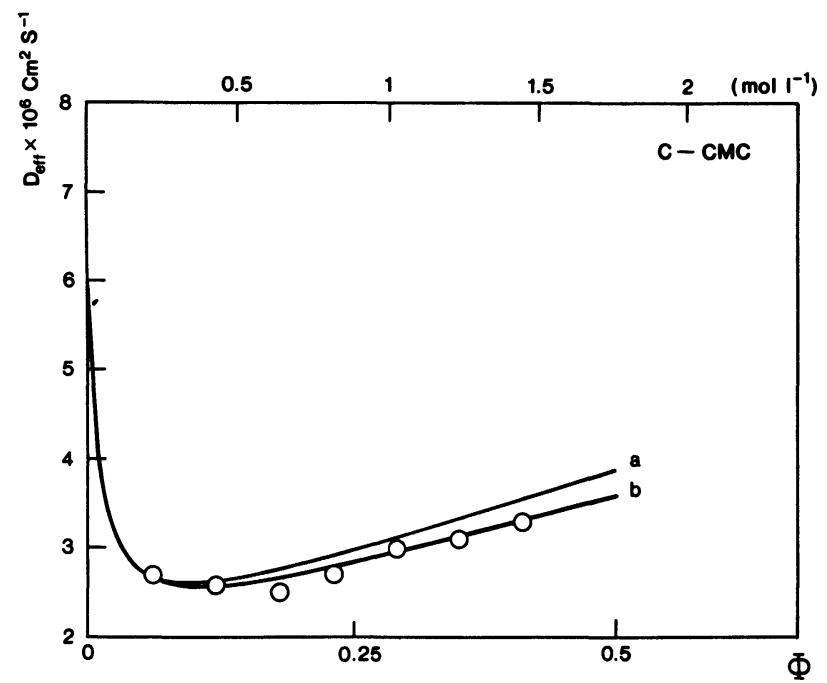

Fig. 2. - Effective diffusion coefficient $D_{\text {eff }}$ versus the volume fraction $\phi_{\mathrm{HS}}$ of S.Oct. $\mathrm{O}$ : experimental data. Full line : theoretical curve with $D_{1}^{0}=1.95 \times 10^{-6} \mathrm{~cm}^{2} / \mathrm{s}, D_{2}^{0}=6 \times 10^{-6} \mathrm{~cm}^{2} / \mathrm{s}, N=12(1+37 \phi)[19]$, (a) $k=2.0 ;(b) k=1.7$.

The good agreement between the calculated curves and the experimental data clearly shows that the observed minimum results from the micelle-monomer exchange. At large volumic fractions the micelle-micelle interaction (thermodynamic and hydrodynamic contribution) dominates the measured values of $D_{\text {eff }}$.

Finally in another paper [19], we considered that Phillies' model [6] could give a qualitative agreement with experimental data of S.Oct. We deduced a " reactive » diffusion constant resulting from the dynamic motion of the micelle during the monomer-micelle exchange. Using this crude calculation, a good agreement with experiment was obtained, but the concentration depen- 
dence of the diffusion coefficient was not used. The present theory gives a quantitative description for two micellar systems. By taking into account the effect of kinetic exchange in micellar solutions, the increase in $D_{\text {eff }}$ near the c.m.c. is well described.

\section{Appendix.}

- A short and elegant method to obtain the effective diffusion coefficient for fast chemical equilibrium between monomers $(\mathrm{m})$ and micelles $(\mathrm{M})$ is the following [23] :

$$
\begin{aligned}
& \mathbf{J}_{\mathrm{m}}=-D_{\mathrm{m}} \nabla C_{\mathrm{m}} \\
& \mathbf{J}_{\mathrm{M}}=-D_{\mathbf{M}} \nabla C_{\mathbf{M}} .
\end{aligned}
$$

At equilibrium :

$$
C_{\mathrm{m}}^{v}=C_{\mathrm{M}} \times K
$$

and

$$
\begin{gathered}
\frac{\nabla C_{\mathrm{m}}}{\frac{C_{\mathrm{m}}}{v}}=\frac{\nabla C_{\mathrm{M}}}{C_{\mathrm{M}}}=\frac{\Delta C}{C_{\mathrm{M}}+\frac{C_{\mathrm{m}}}{v}} \\
\mathbf{J}_{\text {total }}=\mathbf{J}_{\mathrm{m}}+\mathbf{J}_{\mathrm{M}}=-\frac{D_{\mathrm{m}} \frac{C_{\mathrm{m}}}{v}+D_{\mathrm{M}} C_{\mathrm{M}}}{C_{\mathrm{M}}+\frac{C_{\mathrm{m}}}{v}} \nabla C .
\end{gathered}
$$

Thus

$$
D_{\text {eff }}=\frac{D_{1} v N C_{1}^{0}+D_{2} C_{2}^{0}}{v N C_{1}^{0}+C_{2}^{0}}
$$

This formula is similar to equation 8 and applies for the $q \rightarrow 0$ limit.

- For a mixture of two components, an extension of this formalism to large $q$ values is possible using the generalized Ackerson theory $[24,25]$

$$
D_{\text {eff }}=\frac{\sum x_{i} F_{i}(q) D_{i}(q)+H(q)}{\sum \sqrt{x_{i} x_{j} F_{i j}(q) S_{i j}(q)}},
$$

where $D_{\text {eff }}$ is obtained from the first cumulant of the autocorrelation function. $x_{i}, F_{i}, D_{i}, S_{i}$ are the concentration, the form factor, the diffusion coefficient, the static structure factor of species $i$, respectively.

$H(q)$ is the hydrodynamic contribution.

For a mixture of micelles $(i)$ and monomers $(j)$ at the $q \rightarrow 0$ limit :

$$
\begin{gathered}
F_{i i}=\dot{F}_{i} \rightarrow N^{2} \\
F_{i j}=N \quad \text { and } F_{j}=1 \\
x_{i}=C_{1}^{0}, \quad x_{2}=C_{2}^{0}, \quad D_{i}=D_{1}, \quad D_{j}=D_{2}, \\
D_{\text {eff }}=\frac{C_{1}^{0} N^{2} D_{1}+C_{2}^{0} D_{2}+H(\mathrm{o})}{C_{1}^{0} N^{2} S_{11}(0)+2 \sqrt{C_{1}^{0} C_{2}^{0}} N S_{12}(\mathrm{o})+C_{2}^{0} S_{22}(\mathrm{o})} .
\end{gathered}
$$


When there are no interactions $H(0)=0$ and $S_{11}(0)=S_{22}(0)=1, S_{21}=0$. Then

$$
D_{\text {eff }}=\frac{C_{1}^{0} N^{2} D_{1}^{0}+C_{2}^{0} D_{2}^{0}}{C_{1}^{0} N^{2}+C_{2}^{0}}
$$

With hard spheres thermodynamic and hydrodynamic interactions :

$$
\begin{aligned}
S_{11}(0) & \sim 1-80 \\
H(\phi) & \sim N^{2} C_{1}^{0} \frac{3}{4}\left(S_{11}-1\right) D_{1}^{0} \\
D_{\text {eff }} & \sim \frac{D_{1}^{0} N^{2} \frac{C_{1}^{0}}{C_{2}^{0}}(1-6 \phi)+D_{2}^{0}}{N^{2} \frac{C_{1}^{0}}{C_{2}^{0}}(1-8 \phi)+1} .
\end{aligned}
$$

Near the c.m.c. : $D_{\text {eff }} \sim D_{2}^{0}$.

At large volumic fractions with $N$ constant : $D_{\text {eff }} \sim D_{1}^{0}(1+2 \phi)$.

\section{Acknowledgments.}

We wish to thank L. Belloni for helpful comments.

\section{References}

[1] Lindman, B., Brun, B., J. Coll. Interf. Sc. 422 (1978) 388.

[2] TaYlor, G., Proc. R. Soc. London Ser. A 219 (1953) 1886.

[3] ARIS, R., Proc. R. Soc. London Ser. A 235 (1956) 67.

[4] Corti, M., Degiorgio, V., J. Phys. Chem. 85 (1981) 711.

[5] Dorshow, R., Briggs, J., Bunton, C. A., Nicoli, D. F., J. Phys. Chem. 86 (1982) 2395.

[6] Phillies, G. D., J. Phys. Chem. 85 (1981) 3541.

[7] Evans, D. F., Mitchell, D. J., Ninham, B. W., to be published.

[8] Aniansson, E., Wall, S., Almgren, M., Hoffmann, H., Kielmann, J., Ulbricht, W., Zana, R., Lang, J., Tondre, C., J. Phys. Chem. 80 (1976) 905.

[9] ZANA, R., private communication.

[10] Berne, B. and Pecora, J., Dynamic light scattering (John Wiley and Sons, New York) 1976.

[11] Robinson, R. A. and Stokes, R. Y., Electrolyte solutions.

[12] Hansen, J. P. and Hayter, J., Molec. Phys. 46 (1982) 65.

[13] Hess, W. and KLein, R., Physica 94A (1978) 71.

[14] Kirkwood, J. G. and Buff, F. P., J. Chem. Phys. 19 (1951) 774.

[15] Rice, S. A., Nagasawa, M. and Morawetz, H., Polyelectrolyte solution (Molecular Biology Series Academic Press) 1961.

[16] Rasaiah, J. C. and Friedman, H. L., J. Chem. Phys. 48 (1968) 2742.

[17] Zemb, T., Chachaty, C., Chem. Phys. Lett. 88 (1982) 68.

[18] Tivant, P., Turq, P., Drifford, M., Magdelena, A., Menez, R., Biopolym. 22 (1983) 643.

[19] Zemb, T., Drifford, M., Hayoun, M., Jehanno, A., in press in J. Phys. Chem.

[20] Lindman, B., Wennerstrom,.H., T-opics in Current chemistry, vol. 87 (Springer-Verlag Berlin) 1980.

[21] Hayter, J., Zemb, T., Chem. Phys.'Lett. 93 (1982) 91.

[22] Ekwall, P., Eikrem, H., Mandell, L., Acta Chem. Scand. 17 (1963) 8.

[23] BRochard, F., private communication.

[24] Ackerson, B. J., J. Chem. Phys. 64 (1976) 242.

[25] Belloni, L., Thèse 3e cycle, Paris (1982). 